\title{
Title: Ultra-stable laser interferometry for earthquake detection with terrestrial and submarine optical cables
}

Short title: Submarine optical cables for earthquake detection

\section{Authors:}

Giuseppe Marra $^{1 *}$, Cecilia Clivati ${ }^{2}$, Luckett Richard ${ }^{3}$, Anna Tampellini ${ }^{2,4}$, Jochen Kronjäger ${ }^{1}$, Louise Wright ${ }^{1}$, Alberto Mura ${ }^{2}$, Filippo Levi², Stephen Robinson ${ }^{1}$, André Xuereb ${ }^{5}$, Brian Baptie ${ }^{3}$ and Davide Calonico ${ }^{2}$

\section{Affiliations:}

${ }^{1}$ National Physical Laboratory, Hampton Road, Teddington, TW11 0LW, UK.

${ }^{2}$ I.N.Ri.M., Istituto Nazionale di Ricerca Metrologica, Strada delle Cacce 91, 10135 Turin, Italy. ${ }^{3}$ British Geological Survey, The Lyell Centre, Research Avenue South, Edinburgh, Scotland, UK, EH14 4AP.

${ }^{4}$ Politecnico di Torino, Corso Duca degli Abruzzi 24, 10129, Turin, Italy

${ }^{5}$ Department of Physics, University of Malta, Msida MSD 2080, Malta.

\section{*Correspondence to: giuseppe.marra@npl.co.uk}

One sentence summary: We detected earthquakes on the sea floor measuring seismicallyinduced optical changes in ordinary submarine telecommunications cables. 
Abstract: Detecting ocean-floor seismic activity is crucial for our understanding of the interior structure and dynamic behavior of the Earth. However, $70 \%$ of the planet's surface is covered by water and seismometers coverage is limited to a handful of permanent ocean bottom stations. We show that existing telecommunication optical fiber cables can detect seismic events when combined with state-of-the-art frequency metrology techniques by using the fiber itself as the sensing element. We detected earthquakes over terrestrial and submarine links with length ranging from 75 to $535 \mathrm{~km}$ and a geographical distance from the earthquake's epicenter ranging from 25 to $18,500 \mathrm{~km}$. Implementing a global seismic network for real-time detection of underwater earthquakes requires applying the proposed technique to the existing extensive submarine optical fiber network.

Main text:

Whilst 70\% of the Earth's surface is covered with water almost all seismic stations are on land. Underwater earthquakes of small intensity (moment magnitude $\mathrm{Mw}<4$ ) remain largely undetected as they are too weak to be measured by land-based seismic networks. This limits our ability to identify the source mechanisms of underwater seismic events and our understanding of the internal structure of the Earth.

Underwater seismic sensors, such as Ocean Bottom Seismometers (OBS), have been widely used to study the physics of the Earth (1), from earthquake dynamics to changes in volcanic structure (2), magma generation and mid-ocean ridge development (3). These devices are deployed over geographically limited areas for temporary surveys with data retrieved at the end of the campaign (4-6). Japan, U.S.A. and Canada, have installed permanent arrays of OBSs close to earthquakeprone areas for research purposes and as tsunami alert systems (7-10). However, a permanent array of wired OBSs large enough to cover the Earth's waters would be extremely expensive to 
install. Several more affordable solutions have been proposed $(11,12)$, including potentially adding sensors in future submarine telecommunication repeaters (13). However, the existing submarine telecommunication network itself is a very attractive option for a global, real time real-time seismic network if the fiber itself is used as the sensing element. Such fiber-based network should complement existing land-based seismometer and OBS networks, extending the coverage of underwater earthquakes monitoring.

Submarine optical fiber cables are the backbone of international and intercontinental telecommunication. Since the first installations in the 1990s, the number of links has increased exponentially due to growth in the Internet and mobile services. The current total length of submarine fiber cables is over 1 million km. In 2016 alone approximately 100,000 km of cable were added to the existing network and another 200,000 km planned by mid-2018 (14) (Fig. 1A). Optical fibers can detect seismic events over km-scale links using distributed acoustic sensing techniques (DAS) $(15,16)$. The techniques were developed by the oil and gas industry where they are currently primarily in use. DAS systems uses back-scatter and reflections of the injected optical signal to extract information about local perturbations along the fiber. Because of the non-zero optical losses of the fiber, the signal-to-noise ratio of the returned signal decreases with the travel distance, currently limiting the usable range of this technology to less than $100 \mathrm{~km}$. The feasibility of extending the DAS range to 1000s of km by using optical amplifiers along the link is yet to be demonstrated. Frequency metrology interferometric techniques can overcome the limitation of DAS. These techniques were initially developed by National Measurement Institutes (NMI) for the comparison of next-generation atomic clocks. Metrological optical links up to 2,200 km long already connect some of the largest NMIs in Europe and network expansion is underway (17-20). Fiber links are usually installed in underground utility ducts, such as power or gas lines, or along motorways and are thus exposed to environmental noise. The induced noise is detrimental to 
atomic clock comparisons and suppressed using active cancellation techniques (21). However, we can exploit the sensitivity to environmentally-induced perturbations to detect seismic waves, vibration, and any other sources of other acoustic noise. We can measure changes as small as a few femtoseconds in the propagation delay experienced by the laser light travelling in the fiber with these interferometric techniques. This corresponds to $\sim \mu \mathrm{m}$ length changes that can be measured over lengths of fiber up to several thousands of km. We achieve this level of sensitivity in just $1 \mathrm{~s}$ of measurement time using laser stabilized to state-of-the-art Fabry-Pérot cavities made of ultra-low expansion (ULE) glass (22). Metrology-grade lasers generate phase-stable light over the entire propagation time through the fiber, which ensures propagation time changes are attributed exclusively to the fiber.

Our experiments use light from a ULE-cavity stabilized laser which we have injected at one end of a standard terrestrial or submarine optical link that consists of a fiber pair, one fiber used for each direction of propagation (Fig. 1B). The two fibers are connected at the far end of the optical link to form a loop such that the light returns to the transmitter after a round trip. We combined the injected and returned optical signals on a photodetector and measured their phase difference. The seismically-induced phase changes of the returned optical signal detect local and remote earthquakes.

On 24 ${ }^{\text {th }}$ August 2016 an earthquake of moment magnitude (Mw) 6.0 struck in Central Italy, followed by two more events of Mw 5.9 and Mw 6.5 on $26^{\text {th }}$ and $30^{\text {th }}$ October (23). These events were detected at the National Physical Laboratory (NPL) in Teddington (UK) whilst running frequency metrology experiments on an optical fiber link not intentionally designed to detect seismic waves. This 79 km-long fiber link (UK-L1) connects the National Physical Laboratory in Teddington (UK) to a data center in the nearby town of Reading and is located at a geographical 
distance of approximately 1,400 km from the epicenter of the Central Italy earthquake. The phase fluctuations induced by the seismic event on the laser light propagating in the fiber link for the $30^{\text {th }}$ October event are shown in Fig. 2A and compared to data from a seismic station (Swindon, GB.SWN1) located approximately $100 \mathrm{~km}$ away from the NPL end of the fiber link. The low sampling rate (1 sample/s) prevented us from constraining the magnitude of the primary wave (Pwave) that has a frequency spectrum extending to a few $\mathrm{Hz}$. We detected several other teleseismic events with independently determined magnitudes of Mw 5.9 to 7.9 with epicenters in New Zealand, Japan and Mexico. We achieved a higher signal-to-noise ratio on another 75 km-long optical link (UK-L2) in South-East England in late 2017. This link runs almost entirely in nonmetropolitan areas, which resulted in lower environmentally-induced noise levels as compared to UK-L1. At the same time, the Istituto Nazionale di Ricerca Metrologica (INRIM) in Turin (Italy) established a 535 km link (IT-L1) between Turin and Medicina near the town of Bologna. The optical phase sampling rate for UK-L2 and IT-L1 was 100 samples/s. On $12^{\text {th }}$ November 2017 we detected the Mw 7.3 earthquake on the Iraq-Iran border with both UK-L2 and IT-L1 links (Fig. 2B). We determined the arrival times for the $\mathrm{P}$ and $\mathrm{S}$ waves using the UK-L2 link, which were consistent with the first arrivals identified using the nearby seismic station at Herstmonceux (GB.HMNX). Periodic environmental perturbations in the IT-L1 link made it difficult to resolve the P-wave, but the following seismic perturbations were clearly visible.

The detection sensitivity of a terrestrial optical fiber link, like for seismometers, is primarily limited by surrounding man-made noise in the frequency range of interest for earthquake detection (0.1 to $20 \mathrm{~Hz}$ ). We expected substantially lower background noise per unit length over submarine optical links. We conducted metrology experiments with an ultra-stable laser source on a submarine link (IT-L2) in September 2017. During a two-day measurement campaign on the 96.4 km-long submarine cable between Malta and Sicily we detected a local magnitude (ML) 3.4 
earthquake, with an epicenter $85 \mathrm{~km}$ away in the Malta Sea (Fig. 3A). We measured the optical phase perturbation and compared it to the displacement recorded by seismometers located within a few km of each end of the fiber link (MN.WDD, Malta and IV.HPAC, Sicily) (Fig. 3A). We observed a delay of approximately $2 \mathrm{~s}$ between the P-wave detected by the link and the MN.WDD station. This delay is consistent with the travel distance between seismometer and the Malta end of the fiber link at a speed of approximately $5 \mathrm{~km} / \mathrm{s}$, which we calculated from the delay observed between the MN.WDD and IV.HPAC seismograms. We clearly identified both P and S waves. We measured the root mean square (RMS) level of environmental noise of the IT-L2 submarine link to be 8 and 5 times lower than the UK-L1 and UK-L2 links respectively in the frequency range 0.1 to $20 \mathrm{~Hz}$. In coastal areas the ambient noise in this frequency range arises primarily from commercial shipping, local wind-sea, wave-wave and wave-shore interactions. We expect a quieter environment for cables resting on the ocean floor in much deeper waters ( $\sim \mathrm{kms})$ than the shallow depth (200 m) of the busy Malta-Sicily channel, allowing the detection of low magnitude earthquakes on substantially longer links than IT-L2 (24). Submarine cables cross several seismically active areas, such as the North and Mid-Atlantic ridge, the South American, North American, and African Plates triple junction. Seismic monitoring of all these areas relies almost entirely on land-based seismic stations. Earthquakes of magnitude lower than 4 are largely undetected as they are too weak by the time they reach seismometers on the nearest island or mainland. Such earthquakes typically are detected only up to a few hundred km from the epicenter, and would affect a relatively small fraction of the submarine fiber links. A similar scenario, on a smaller scale, occurred for detection of the Mw 4.4 Parma (Italy) earthquake on the IT-L1 link in November 2017 that was also detected by nearby seismometers (Fig 3B). Here, the epicenter was $25 \mathrm{~km}$ away from the nearest section of the $535 \mathrm{~km}$-long fiber link. We identified the time of arrival of the detected seismic wave, which corresponded to the smaller distance between the 
epicenter and the fiber link. This also leads to the highest amplitude of the detected signal, as confirmed by the seismometer traces.

We can determine the point at which the seismic wave reaches a fiber link by transmitting the laser light in both directions in a standard telecommunication fiber pair, as depicted (Fig 4A). We can measure the distance travelled along the fiber by cross-correlating the seismic signals recorded at each end of the link with a high-speed phase sampler. We used this technique in the laboratory to demonstrate proof-of-concept where we were able to identify the location of an environmental perturbation to within $1 \mathrm{~km}$ over $101 \mathrm{~km}$ of spooled fiber (24). Two fiber links, following different paths, allows determination of the epicenter (Fig. 4B). The exact route of each fiber, required to calculate the epicenter, is normally known to within $1 \mathrm{~km}$. We note that two line sensors (the links) eliminate the need for a third node to triangulate, as traditional seismology requires. Additional links improve the epicenter location accuracy.

In contrast to seismometers, optical fibers are sensitive to seismic perturbations over their entire length rather than at a single point in space. The detected signal will be the result of integration of these perturbations. However, for earthquakes whose epicenter is at a distance shorter than the length of the link (as it would be in the case of small local earthquakes detected by trans-oceanic links), sections of the fiber far from the first point of contact of the seismic wave with the link will not contribute significantly to the detected signal, owing to the attenuation of the seismic wave with distance (this can be inferred, for example, from the seismometer traces in Fig. 3B for the Parma earthquake). The arrival time of the P-wave, one of the most important parameters for earthquake characterization, is not significantly affected by the distributed nature of the detection as only a small section of the link is perturbed upon its arrival. Also unlike seismometers, the signal detected using optical fiber links is the result of the superimposition of perturbations along the 
three components of motion. Whilst the resulting signal from the distributed detection with optical fiber might require different analysis than that performed on seismometer data, crucial information can still be extracted and can prove invaluable from locations where no data can currently be obtained otherwise.

We used several optical fibers installations to detect local earthquakes and teleseisms, along with presenting a strategy for using the existing submarine telecommunications optical fiber infrastructure. Using existing cables should provide a cost-effective complement to ocean bottom seismometers and further advance our understanding of the dynamic behavior of Earth's interior. The phase stability of metrology-grade lasers is sufficient to enable coherent measurements over fiber lengths well beyond 10,000 km, enabling measurement on trans-oceanic links. Fiber optic earthquake detection may be the preferred option for more remote areas like the Arctic sea (25). We anticipate that submarine fiber networks could also be used for applications beyond seismic monitoring, from marine mammal migration tracking (26) to sea noise pollution monitoring, a growing matter of concern world-wide for its impact on marine life $(27,28)$.

\section{References and notes}

1. Y. Kaneda, Introduction to ocean floor networks and their scientific application, Marine Geophysical Research 35, 177-180 (2014).

2. D. R. Toomey et al. The cascadia initiative: A Sea Change In Seismological Studies of Subduction Zones, Oceanography 27, 138-150 (2014).

3. D. W. Forsyth et al. Imaging the deep seismic structure beneath a mid-ocean ridge: The MELT experiment, Science 280 (1998).

4. Ocean Bottom Seismograph Instrument Pool (OBSIP), http://www.obsip.org 
5. H. Nakstad, J.T. Kringlebotn, Realisation of a full-scale fiber-optic ocean bottom seismic system. Proceedings, 19th International Conference on Optical Fiber Sensors 7004, 700436 (2008).

6. G. A. Cranch, P. J. Nash, C.K. Kirkendall, Large-scale remotely interrogated arrays of fiber-optic interferometric sensors for underwater acoustic applications. IEEE Sensors Journal 3, 19-30 (2003).

7. B. Romanowicz. et al. The Monterey bay broadband ocean bottom seismic observatory. Annals of Geophysics 49, 2-3 (2006).

8. The Ocean Observatories Initiative. http://oceanobservatories.org/.

9. Dense Oceanfloor Network system for Earthquakes and Tsunamis https://www.jamstec.go.jp/donet/e/rd/index.html

10. North East Pacific Time-series Underwater Networked Experiments (NEPTUNE). http://www.oceannetworks.ca/observatories/pacific

11. J. Berger, G. Laske, J. Babcock, J. Orcutt, An ocean bottom seismic observatory with near real-time telemetry, Earth and Space Science 3, 68-77 (2016)

12. Y. Hello, A. Ogé, A. Sukhovich, G. Nolet, Modern Mermaids: New Floats Image the Deep Earth. Earth and Space Science, Transactions American Geophysical Union 92, 337-338 (2011).

13. ITU/WMO/UNESCO IOC Joint Task Force. https://www.itu.int/en/ITU-T/climatechange/task-forcesc/Pages/default.aspx

14. Submarine Telecoms Industry Report. http://subtelforum.com/Report5/D2C63B44C07ABF9C60E4BE6591014FCE/IndustryReport5.pdf

15. J. Mestayer et al., Field trials of distributed acoustic sensing for geophysical monitorings, SEG Technical Program Expanded Abstracts, 4253-4257 (2011).

16. N. J. Lindsey et al., Fiber-Optic Network Observations of Earthquake Wavefields, Geophysical Research Letters, Early view article DOI 10.1002/2017GL075722 (2017).

17. P. Delva et al., Test of Special Relativity Using a Fiber Network of Optical clocks. Phyical Review Letters 118, 221102 (2017). 
18. C. Lisdat et al., A clock network for geodesy and fundamental science. Nature Communications 7, 12443 (2016).

19. S. Droste et al., Optical Frequency Transfer over a Single-Span 1840 km Fiber Link. Physical Review Letters 111, 110801 (2013).

20. C. Clivati et al., A VLBI experiment using a remote atomic clock via a coherent fiber link. Scientific Reports 7, 40992 (2017)

21. S. M. Foreman, K. W. Holman, D. D. Hudson, D. J. Jones, J. Ye, Remote transfer of ultrastable frequency references via fiber networks. Rev. Sci. Instrum. 78, 021101 (2007).

22. K. Numata, A. Kemery, J. Camp, Thermal-Noise Limit in the Frequency Stabilization of Lasers with Rigid Cavities. Physical Review Letters 93, 250602 (2004).

23. L. Chiaraluce et al., The 2016 Central Italy Seismic Sequence: A First Look at the Mainshocks, Aftershocks, and Source Models, Seismological Research Letters 88, 757-771 (2017).

24. Supplementary materials, Material and Methods section

25. J. Hsu, An Internet cable will soon cross the Arctic. Scientific American, https://www.scientificamerican.com/article/an-internet-cable-will-soon-cross-the-arctic-circle/ (2016)

26. N. E. Balcazar et al., Using calls as an indicator for Antarctic blue whale occurrence and distribution across the southwest Pacific and southeast Indian Oceans. Marine Mammal Science, 33, 172-186 (2017).

27. I. L. Boyd et al. An international quiet ocean experiment. Oceanography 24, 174-181 (2011).

28. J. L. Miksis-Olds, S. M Nichols, Is low frequency ocean sound increasing globally?, J. Acoust. Soc. Am. 139 (1) 501-511, (2016).

Acknowledgements The UK side of this work was funded by the Department for Business, Energy and Industrial Strategy (BEIS) as part of the UK National Measurement System program. The UK-L1 fiber link was funded by the UK Space Agency. INRIM acknowledges for funding the Italian Ministry of Education, University and Research (MIUR) through the Progetti Premiali 
2014 and 2015 programs (LABMED and METGESP projects). We acknowledge funding by the Research, Innovation and Development Trust of the University of Malta. The British Geological Survey contribution to this work is funded by the Natural Environment Research Council (NERC). We are very grateful to Melita Limited and Enemalta plc for providing us with access to the submarine fiber links. We thank Prof. Massimo Inguscio, President of the Consiglio Nazionale delle Ricerche (CNR), for supporting and encouraging the Italy-Malta experiment. We thank Helen Margolis (NPL), Salvatore Micalizio (INRIM) and Prof. Pauline Galea (University of Malta) for fruitful discussions. The facilities of IRIS Data Services, and specifically the IRIS Data Management Center, were used for access to waveforms, related metadata, and/or derived products used in this study. IRIS Data Services are funded through the Seismological Facilities for the Advancement of Geoscience and EarthScope (SAGE) Proposal of the National Science Foundation under Cooperative Agreement EAR-1261681.

G.M planned, designed and conducted the experiments on the UK links and prepared the first draft of the manuscript. R.L. and B.B. analysed the seismic data and provided seismology expertise. D.C. and F.L. planned and designed the experiments on IT-L1 and IT-L2. C.C., A.M., F.L. and A.T. conducted the experiments on the IT-L1 link. C.C., D.C., A. X., and A.T. conducted the experiments on the IT-L2 link. L.W. developed the analytical tools. J.K. set up the UK-L2 link. S.R. provided underwater acoustic expertise. All authors contributed extensively to the discussion, interpretation of the data and manuscript preparation.

Optical phase data is available in the supplementary materials. 
Fig. 1. (A) Submarine telecommunication cable map. Illustration of the existing and planned submarine telecommunication infrastructure. Optical frequency metrology techniques enable these fiber links to be used for the detection of earthquakes at the bottom of seas and oceans. Map data (C) OpenStreetMap contributors; Cable data: TeleGeography’s Telecom Resources licensed under Creative Commons Share alike. (B) Illustration of the optical setup used in our experiments for measuring the seismically-induced perturbation of the optical signal travelling in the fiber. The same principle was used for terrestrial and submarine fiber links (only the latter case is illustrated in the figure). ULE: ultra-low expansion glass used to stabilize the laser frequency.

Fig. 2. Tele-seismic events on terrestrial optical links. (A) Comparison between the seismicallyinduced optical phase changes detected on the UK-L1 link and the signal from a seismometer in Swindon (GB.SWN1) for the Central Italy earthquake on $30^{\text {th }}$ October 2016. (B) Comparison between the phase changes detected on the IT-L1 and UK-L2 link with the signals from seismometers in Monterenzio (MN.MTRZ) and Herstmonceux (GB.HMNX) for the Iraq border earthquake on $12^{\text {th }}$ November 2017. The north-south component has been used for all seismic station data.

Fig. 3. Small magnitude earthquakes on submarine and terrestrial link. (A) Seismic wave detected on the submarine IT-L2 link for the Malta Sea earthquake on $2^{\text {nd }}$ September 2017 compared with signals from seismometers located within a few $\mathrm{km}$ of each end of the link. High pass filtering at $1.5 \mathrm{~Hz}$ has been applied to the optical signal to suppress a strong environmentally-induced $1 \mathrm{~Hz}$ component that was present on the optical signal. The same filter has been applied on the signals from seismic station. (B) Comparison between the phase changes 
detected on IT-L1 link and seismometers located close to four intermediate points along the link for the Parma earthquake on $19^{\text {th }}$ November 2017. The north-south component has been used for all seismic station data.

Figure 4 | Seismic event localization techniques. (A) Because of the finite propagation speed of the light in the fiber $\left(\sim 2 \times 10^{8} \mathrm{~m} / \mathrm{s}\right)$ seismically-induced optical phase perturbations will reach the two ends of a bidirectional fiber link at different times. The location on the optical fiber link at which the seismic wave first reaches it can be determined by calculating the delay difference by cross-correlating the received signals. Earthquakes located on either side of the link and along the perpendicular to the link axis will result in the same identified location along the fiber. By using two links the location ambiguity can be resolved. (B), Localization of the epicenter using two bidirectional fiber links. Simple geometry allows the coordinates of the epicenter to be found from the location of the point of first contact of the seismic wave along the fiber. TX: ultra-stable laser injecting light into the fiber. RX: optical detection and phase comparison unit. This unit measures the optical phase difference between the light generated by the local TX laser and that transmitted through the fiber link by the remote TX laser. 